\title{
Molecular Imaging of Carotid Plaque Vulnerability
}

\author{
Angelika Alonso Dimitrios Artemis Michael G. Hennerici \\ Department of Neurology, Universitätsmedizin Mannheim, University of Heidelberg, Germany
}

\section{Key Words}

Carotid artery disease $\cdot$ Molecular imaging $\cdot$ Plaque

vulnerability $\cdot$ Contrast-enhanced ultrasound .

Neovascularization

\begin{abstract}
Background: Carotid endarterectomy (CEA) has been shown to be beneficial in patients with high-grade symptomatic carotid artery stenosis. Patients with high-grade asymptomatic stenosis may only exceptionally benefit from CEA during periods of increased plaque vulnerability. Imaging modalities to characterize unstable, vulnerable plaques are strongly needed for better risk stratification in these patients. Summary: Contrast-enhanced ultrasound (CEUS) is a novel and noninvasive technique capable to identify several surrogate markers of vulnerable carotid plaques. The use of specific ultrasound microbubbles allows a reliable detection of microulcerations due to an optimized visualization of the plaque-lumen border. As microbubbles are strictly intravascular tracers, the detection of individual microbubbles within the plaque corresponds to intraplaque neovessels. The accuracy of CEUS in the visualization of newly formed microvessels has been confirmed in histological studies on carotid endarterectomy specimens. Together with the formation of adventitial vasa vasorum, intraplaque neovascularization is a strong predictor for symptomatic disease. The phenomenon of late phase contrast enhancement is based on the adherence of microbubble-containing monocytes on inflamed endothelium. Recent studies suggest that late phase contrast enhancement may reflect endothelial inflam-
\end{abstract}

mation or activation within carotid plaques. The development of conjugated microbubbles that bind to specific ligands such as thrombotic material or neovessels has led to the term 'molecular imaging'. CEUS with microbubbles targeted to P-selectin and VCAM-1, key molecules in leukocyte trafficking, was used to detect an inflammatory plaque phenotype, whereas microbubbles coupled to the VEGF-receptor may allow for a detection of neovascularization. Even though imaging with targeted microbubbles is yet in an experimental stage, this technique can visualize active plaque reorganization with increased vulnerability leading to generation of arterio-arterial embolism. Key Messages: The use of contrast-enhanced ultrasound can be recommended to assess atherosclerotic carotid lesions at risk for rupture. Prospective clinical studies are needed to validate the use of CEUS in patients with high risks of recurrent large artery strokes. In particular, this applies to the detection of intraplaque neovascularization, a well-established marker in preclinical and observational studies, while the clinical significance of late phase contrast enhancement still needs to be determined.

(c) 2014 S. Karger AG, Basel

\section{Introduction}

Although more than 60 years have passed since 1951, when C. Miller Fisher reported in his milestone work the occurrence of transient ischemic attacks (TIA) in relation to carotid artery disease, reliable criteria to identify patients with high-risk carotid plaques are still lacking. To

\section{KARGER 125}

() 2014 S. Karger AG, Base

1015-9770/14/0391-0005\$39.50/0

E-Mail karger@karger.com

www.karger.com/ced 
date, the degree of carotid artery stenosis remains the best evaluated marker for the risk of stroke in carotid artery disease. Especially after the two multi-center trials of the 90's, that is, NASCET [1] and ECST [2], in clinical practice, the management of patients with carotid artery plaques highly depends on the grade of stenosis. As carotid duplex evaluation of the carotid atherosclerotic disease has become an essential component in the diagnosis and management of carotid artery disease [3], many different ultrasound plaque parameters were postulated as possible predictors of increased risk of stroke in patients with carotid artery disease. Extensive work on atherosclerotic plaque specimens from carotid endarterectomy consistently demonstrated that the individual composition of the plaque is a major factor determining the risk of cerebral ischemia $[4,5]$. From a pathophysiological viewpoint, the transition from a stable to an unstable atherosclerotic plaque is a consequence of complex molecular and cellular mechanisms. Vulnerable plaques, which are prone to rupture, characteristically have a large lipid necrotic core [6] and are covered by a thin fibrous cap [7]. In vulnerable plaques, the number of smooth muscle cells (SMC) is decreased through the induction of SMC apoptosis, mainly by inflammatory macrophages and lymphocytes. As a consequence, the extracellular matrix components secreted by SMC decrease, and the fibrous cap thins out. Apoptosis of macrophages further contributes to fibrous cap thinning and enlargement of the necrotic core. The effective clearance of apoptotic cells by phagocytes is impaired, resulting in the accumulation of apoptotic cells and decreased anti-inflammatory impact of the phagocytes. In a growing plaque, reduced oxygen diffusion results in hypoxia and in the release of angiogenic growth factors. Intraplaque neovessels with immature structure are formed. The absence of pericytes in these vessels may lead to increased leakage and predisposes the vessels to rupture.

To date, plaque neovascularization is considered to represent a most important predictor of carotid plaque vulnerability. Several studies on endarterectomy specimens linked the presence of intraplaque neovessels and subsequent intraplaque hemorrhage to symptomatic periods of otherwise long-lasting asymptomatic stages of carotid artery disease $[8,9]$. This review updates our current knowledge about the potential of contrast-enhanced ultrasound in the detection of vulnerable plaques in these patients. We will give an overview of the current status of CEUS, features of vulnerable plaques accessible to ultrasound, the role of CEUS in comparison to MR imaging modalities, and the future perspectives in carotid plaque imaging.

\section{Contrast Agents}

Contrast-enhanced ultrasound is based on an ultrasound-specific contrast agent consisting of microbubbles and was initially used as early as 1968 by Gramiak and Shah in echocardiography [10]. Due to their size, microbubbles cannot leave the capillary bed and can thus serve as strictly intravascular tracers. The echogenicity of microbubbles results from the high impedance contrast as against the liquid in which they are expanded and their ability to absorb and re-radiate sound energy. The re-radiated sound field thus comprises different harmonics of the excitation frequency, making the microbubble signal distinguishable from the surrounding tissue signals [11]. The first-generation microbubbles were room air microspheres with no ability to cross the pulmonary bed and therefore useless to carotid ultrasound imaging. The clinical relevance of vascular ultrasound led to the development of echocontrast agents able to survive pulmonary and capillary transit and increase the echogenicity of the flowing blood [12]. The in vivo stability of the second generation microbubbles is related to their shell characteristics and to the solubility in blood of the gas used in their preparation. The second generation of microbubbles contains either phospholipid-encapsulated sulphur hexafluoride or albumine- or lipid-encoated perfluorpentan. These microbubbles have, due to the low solubility of the gas that they contain and due to their stabilized shell, a high circulation time.

Commercially available microbubbles have been already used for diagnostic purposes in millions of patients and are considered safe. The reported side effects seem to be extremely rare. However, there are some contraindications that should be considered. Those are known allergy to the contrast agent; unstable angina pectoris; myocardial infarction; acute cardiac failure, significant cardiac shunt and endocarditis [13]. Currently, four second-generation microbubbles are approved and used in clinical practice: Optison (perflutren protein type-A microspheres), Definity (perflutren lipid microspheres), Sono Vue (phospholipid-encapsulated sulphur hexafluoride microbubbles), and Levovist (granules composed of galactose and palmitic acid). To date, microbubble contrast agents are routinely used in neurosonology with transcranial color-coded ultrasonography to overcome an insufficient transtemporal window [14]. However, the contrast agents have not been approved for carotid ultrasound examination to this point. Nonetheless, the 2011 guidelines for nonhepatic applications of CEUS by the European Federation of Societies for Ultrasound in Medicine and 


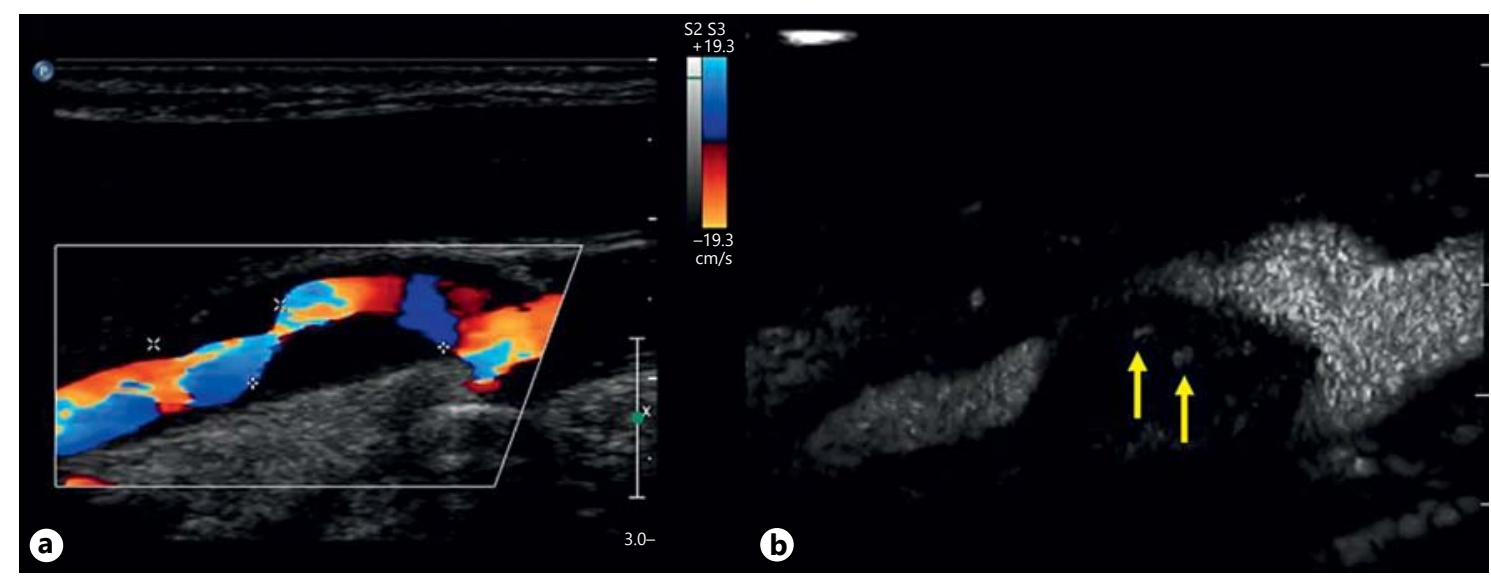

Fig. 1. Identification of intraplaque neovascularization with contrast-enhanced ultrasound. Color-coded duplex of the proximal internal carotid artery with detection of a high-grade stenosis with hypoechoic atherosclerotic plaque (a). The corresponding CEUS scan (b) shows punctual contrast enhancement in the plaque (see arrows), consistent with intraplaque neovessels.

Biology recommend the use of microbubbles for improved lumen delineation and for the differentiation of carotid artery occlusion and characterization of high grade stenosis [15].

\section{Carotid Plaque Imaging with CEUS}

The carotid intima-media-thickness (CIMT) has been validated as a surrogate marker of atherosclerosis in various clinical trials. Conventional ultrasound imaging is considered to be the method of choice for CIMT measurement [16]. Some years ago, Rajaram and colleagues could show that the delineation of the carotid near wall and the associated near wall CIMT can be improved with the use of ultrasound contrast agents [17]. However, this method has not been included in daily practice, because of the high accuracy and reproducibility of CIMT measurement in the far wall even with non-enhanced ultrasound.

CIMT and carotid artery plaque should be considered distinct phenotypes of atherosclerosis. Carotid plaque imaging has largely improved with introduction of CEUS, making use of two mechanisms: first, enhancement of the carotid lumen and characterization of plaque morphology, and second, detection of intraplaque neovascularization and adventitial vasa vasorum.

Carotid plaque ulceration is a strong predictor of symptomatic disease and cerebrovascular events. However, several studies demonstrated that conventional ultrasound is unreliable in detection of ulcerated plaques
$[18,19]$. In a recently published prospective study, the value of CEUS for the detection of carotid plaque ulceration in patients with symptomatic carotid artery disease was investigated. First, the plaque border was visually scored as smooth, irregular, or ulcerated by two readers. Then, disruptions of the plaque-lumen-border were analyzed, with disruptions of $\geq 1 \times 1 \mathrm{~mm}$ defining carotid plaque ulceration. Computed tomographic angiography of the carotid artery was used as the reference technique. With respect to sensitivity and diagnostic accuracy, CEUS was found to be clearly superior to color Doppler ultrasound [20].

The presence of newly generated blood vessels within atherosclerotic lesions is a novel marker of plaque vulnerability and has been associated with symptomatic carotid disease [21]. Development of adventitial vasa vasorum, finally enveloping the plaque, is a further hallmark in later stages of plaque growth. Feinstein was the first to report in 2006 the in vivo identification of adventitial vasa vasorum and neovascularization in the carotid atherosclerotic plaque, although no histologic confirmation was presented in this study [22]. The capability to depict intraplaque neovascularization is based on the high resolution and echocontrast, enabling the detection of individual microbubbles in the vascular bed. As microbubbles are strictly intravascular tracers, the detection of microbubbles within the plaque complies with intraplaque neovessels (for an example, see fig. 1 and online suppl. video, www.karger.com/doi/10.1159/000369123).

Since then, a number of investigators reported a histological validation of the examination. A higher amount of 
contrast enhancement was shown to correlate with an increased density of microvessels in endarterectomy specimens $[23,24]$. In a study with histologic analysis of neovascularization using CD31-stained specimens, the CEUS-determined neovascularization correlated well with the presence of CD31, an integral membrane glycoprotein that is expressed at high levels on endothelial cells. A good correlation with the CEUS results was further shown using a composite of CD34, von Willebrand factor and hemosiderin [25]. A further study linked the presence of neovascularization to inflammatory plaque components. Quantification of neovessels using CEUS was well correlated with the number of inflammatory cells present in the plaque, determined by CD3 and CD68 stains [26]. Moreover, contrast enhancement of carotid plaques correlates strongly with plaque morphology. Ultrasonic plaque echolucency was shown to be associated with an increased risk for stroke in both symptomatic and asymptomatic carotid artery stenosis $[27,28]$. In a series of 52 lesions, a higher degree of contrast enhancement was found in echolucent plaques [23]. Several studies confirmed the occurrence of neovessels in echolucent and mixed plaques $[29,30]$ while neovascularization could not be detected in calcified and echogenic plaques [30]. However, one should bear in mind that these findings are prone to the intrinsic limitations of ultrasound studies such as the acoustic shadowing caused by calcification. For the association of intraplaque neovascularization and degree of stenosis, two studies showed conflicting results. Coli and colleagues reported no correlation between stenosis degree and neovascularization at histology or the grade of contrast-agent enhancement [23]. However, Staub and colleagues found a significantly higher grade of neovascularization in lesions with higher degree of stenosis [31].

The clinical significance of CEUS-detected neovascularization was investigated in a number of studies. In patients with TIA or stroke, a significantly higher contrast enhancement despite a similar plaque thickness was found compared to asymptomatic patients [32]. Vasa vasorum and plaque neovascularization as detected by CEUS were associated with past myocardial infarction and transient ischemic attacks or stroke in an observational study in 147 subjects. The degree of intraplaque neovascularization and vasa vasorum was well correlated with a history of cardiovascular events [33]. In this line, a pilot study in 73 patients undergoing carotid endarterectomy reported a pattern with accumulation of diffuse microbubble contrast at the base of the plaque in all patients with symptomatic carotid artery stenosis, but only in 1/64 asymptomatic patients. In a subsequent histologic analysis of the specimens, the areas with contrast enhancement were characterized by an increased number of microvessels [24]. Taken together, these studies support the role of contrast-enhanced ultrasound imaging of carotid plaques in risk stratification of lesions. Ultrasound-based detection of intraplaque neovascularization is a strong predictor of plaque vulnerability in symptomatic disease.

Recently, late phase contrast enhancement has been investigated as a novel surrogate marker of plaque vulnerability. The phenomenon of late contrast enhancement is based on the phagocytosis of microbubbles by monocytes and subsequent adherence of microbubble-containing monocytes on inflamed endothelium [34]. In addition, microbubbles themselves have also been shown to adhere to the surface of damaged endothelium [35]. Owen and colleagues found that the late phase (six minutes after bolus injection) contrast-enhanced plaque echogenicity was significantly greater in patients with symptomatic carotid artery disease than in those with asymptomatic disease. The authors conclude that late phase contrast enhancement may have the potential to reflect endothelial inflammation or activation within carotid plaques. However, the number of enrolled subjects was small, and the authors reported some overlap in echogenicity between the two groups [36]. Another study by the same group could provide histological evidence for their hypothesis. The authors performed quantitative immunohistochemical analyses for CD68, a macrophage marker, and CD31, an endothelial marker, in carotid endarterectomy specimens. Late enhancement of plaques in preoperative CEUS was significantly correlated with CD68 immunopositivity, indicating an association of late enhancement with inflammation in these plaques. Additionally, a positive correlation of late enhancement with CD31 expression as a surrogate for neovascularization was demonstrated [37]. Further studies in larger cohorts will be needed to confirm the diagnostic value of late enhancement in carotid plaque imaging.

\section{CEUS versus MR Imaging}

Contrast enhanced ultrasound has been proven to be a safe and noninvasive method to assess in real-time atherosclerotic lesions of the carotid arteries. Main advantages of CEUS are the high-resolution imaging; the improved delineation of the vessel wall and plaque morphology, as well the improved detection of anomalies of the 
plaque surface such as ulcerations and micro-ulcerations. The potential to reliably detect neovascularization can be a helpful tool for the clinician in the further risk stratification of patients with carotid atherosclerotic disease. Recently, high-resolution contrast-enhanced MR imaging has attracted some attention in carotid plaque characterization. Quantification of the intact fibrous cap, characterized by contrast enhancement, and the non-enhancing lipid-rich necrotic core in vivo has shown a good correlation with histological findings in endarterectomy specimens [38]. Plaque rupture, a further main characteristic of instable plaques, has successfully been imaged with a three-dimensional TOF bright blood imaging technique [39]. Dynamic contrast-enhanced MR imaging using a kinetic model to estimate the fractional blood volume was performed for quantification of the amount of neovessels in the carotid plaque. The results were highly correlated with the histological quantification in the obtained endarterectomy specimens. However, interstitial volumes with rapid exchange of contrast agent with the plasma could not reliably be distinguished from blood and may thus lead to an overestimation of neovascularization [40].

Systematic comparisons between these two imaging modalities are still lacking. Both methods have their specific limitations and restrictions for clinical applications. MR imaging is time-consuming and limited by many contraindications, while CEUS might be limited by the occurrence of calcified plaques with subsequent acoustic shadowing. Ongoing work concentrates on the value of combining two or more imaging modalities with complementary information [41]. A recently initiated prospective multicenter study was designed to assess carotid plaque characteristics with several imaging modalities including ultrasound and MR imaging in order to identify patients with $<70 \%$ carotid artery stenosis with an increased risk of recurrent stroke [42].

\section{Future Perspectives: Molecular Contrast Ultrasound Imaging}

A substantial limitation of both methods is their application only in advanced stages of atherosclerotic disease. The emerging field of 'molecular imaging', applicable both in ultrasound and MR imaging, offers a new perspective even for early stages of disease.

Ultrasound imaging using second generation microbubbles conjugated with monoclonal antibodies to specific ligands could enhance the diagnostic applications of carotid ultrasound examination. Targeting the molecular basis of plaque formation may allow an early detection of the disease and enable a better risk stratification. The concept of targeting thrombotic material with ultrasound is not new. Lanza et al. provided in 1996 the first in vivo demonstration of a site-specific ultrasonic contrast agent for the intravascular detection of thrombi [43]. Complementary, in vitro studies demonstrated the effectiveness of the targeted microbubbles to bind to a blood clot [44].

In atherosclerotic artery disease, targeted microbubbles have been tested in the assessment of molecular markers in the processes of neoangiogenesis, thrombosis, and inflammation in various animal models. In a mouse model of age-dependent atherosclerosis, molecular imaging with ultrasound was used to detect a lesion-prone vascular phenotype. P-selectin and VCAM-1 are involved in the regulation of leukocyte trafficking, an early step in inflammatory processes involved in plaque formation. Microbubbles targeted to P-selectin and VCAM-1 were shown to bind preferentially to regions of lesion formation [45]. In the same mouse model, CEU molecular imaging with microbubbles targeted to VCAM-1 was performed to monitor the therapeutic effects of statins. The use of statins was associated with less endothelial expression of VCAM-1. Accordingly, signal enhancement by VCAM-1-targeted microbubbles was observed only in non-treated, but not in statin-treated animals [46]. Monitoring of endothelial inflammatory activation by CEUS molecular imaging has made its way to preclinical studies. In a recent study in non-human primates with diet-induced obesity, a progressive increase in endothelial activation over the time could be demonstrated using P-selectin and VCAM-1 targeted microbubbles [47]. The specific binding and accumulation of VCAM-1 targeted microbubbles even under shear flow conditions has been simulated in several in vitro models [48].

At a later stage of the disease, neovascularization is the most investigated parameter for prediction of plaque vulnerability. Molecular imaging with ultrasound has been used for the detection of endothelial ligands associated with angiogenesis. These techniques have been applied in preclinical models of chronic limb ischemia [49] and also for the detection of neovascularization on the abdominal artery plaques in rabbits using VEGF-receptor targeted microbubbles [50]. Data on carotid artery disease are still missing to date.

The interaction of glycoprotein Ib (GPIb) with endothelial von-Willebrand-Factor ( $\mathrm{vWF}$ ) contributes to a prothrombotic state in atherosclerosis [51]. In an ex vivo aortic injury model, attachment of GPIb-targeted microbubbles was found predominantly at the injury site. In 
carotid artery disease, GPIb-targeted microbubbles may therefore be a promising tool to identify a prothrombotic phenotype. Various approaches have been used in order to identify intravascular thrombi using targeted microbubbles. The targeting of platelets using microbubbles conjugated with antibodies against the integrin GPIIb/ IIIa like abciximab or RGD oligopeptides have been tested. Platelet-targeted microbubbles have successfully been used to detect human thrombotic material in a rat model [52-54]. In a recent study, Wang et al. demonstrated that glycoprotein IIb/IIIa-targeted microbubbles specifically bind to activated platelets in vitro and allow real-time molecular imaging of acute arterial thrombosis and the monitoring of the success or failure of pharmacological thrombolysis in vivo in a mouse model [55]. Cyclic RGDmodified microbubbles targeted to GP IIb/IIIa with contrast-enhanced ultrasound were also shown capable of detecting inflammation-activated platelets and thrombosis in large arteries in an in vivo study [56].

\section{Conclusion}

Contrast-enhanced ultrasound is a new imaging tool in the evaluation of carotid artery plaques. An improved characterization of the plaque morphology with the detection of microulcerations and the visualization of intraplaque neovessels as well as adventitial vasa vasorum may help to define a high-risk phenotype during long periods of silent, asymptomatic conditions. An optimized risk stratification is strongly needed to select very few patients considered for carotid endarterectomy prior to clinical events resulting from incidental plaque vulnerability. In addition, best medical treatment concepts may better be developed if matched with evidenced modification of targeted structure development. Molecular contrast ultrasound imaging using microbubbles targeted to endothelial key molecules in plaque formation provides a perspective for the detection of carotid atherosclerosis in the very early stages of the disease.

\section{References}

1 Clinical alert: benefit of carotid endarterectomy for patients with high-grade stenosis of the internal carotid artery. National Institute of Neurological Disorders and Stroke Stroke and Trauma Division. North American Symptomatic Carotid Endarterectomy Trial (NASCET) investigators. Stroke 1991;22: 816-817.

2 Rothwell PM, Gutnikov SA, Warlow CP: Reanalysis of the final results of the European Carotid Surgery Trial. Stroke 2003;34:514523.

-3 Byrnes KR, Ross CB: The current role of carotid duplex ultrasonography in the management of carotid atherosclerosis: foundations and advances. Int J Vasc Med 2012;2012: 187872.

4 Gronholdt ML: Ultrasound and lipoproteins as predictors of lipid-rich, rupture-prone plaques in the carotid artery. Arterioscler Thromb Vasc Biol 1999;19:2-13.

$\checkmark 5$ Fisher M, Paganini-Hill A, Martin A, Cosgrove M, Toole JF, Barnett HJ, Norris J: Carotid plaque pathology: thrombosis, ulceration, and stroke pathogenesis. Stroke 2005; 36:253-257.

6 Mono ML, Karameshev A, Slotboom J, Remonda L, Galimanis A, Jung S, Findling O, De Marchis GM, Luedi R, Kiefer C, Stuker C, Mattle HP, Schroth G, Arnold M, Nedeltchev K, El-Koussy M: Plaque characteristics of asymptomatic carotid stenosis and risk of stroke. Cerebrovasc Dis 2012;34:343-350.

7 Li ZY, Howarth SP, Tang T, Gillard JH: How critical is fibrous cap thickness to carotid plaque stability? A flow-plaque interaction model. Stroke 2006;37:1195-1199.

8 Mofidi R, Crotty TB, McCarthy P, Sheehan SJ, Mehigan D, Keaveny TV: Association between plaque instability, angiogenesis and symptomatic carotid occlusive disease. $\mathrm{Br} \mathrm{J}$ Surg 2001;88:945-950.

-9 Hiyama T, Tanaka T, Endo S, Komine K, Kudo T, Kobayashi H, Shiokawa Y: Angiogenesis in atherosclerotic plaque obtained from carotid endarterectomy: association between symptomatology and plaque morphology. Neurol Med Chir (Tokyo) 2010;50:10561061.

10 Gramiak R, Shah PM: Echocardiography of the aortic root. Invest Radiol 1968;3:356366.

11 Stride E: Physical principles of microbubbles for ultrasound imaging and therapy. Cerebrovasc Dis 2009;27(suppl 2):1-13.

12 Droste DW: Clinical utility of contrast-enhanced ultrasound in neurosonology. Eur Neurol 2008;59(suppl 1):2-8.

13 Main ML, Ryan AC, Davis TE, Albano MP, Kusnetzky LL, Hibberd M: Acute mortality in hospitalized patients undergoing echocardiography with and without an ultrasound contrast agent (multicenter registry results in $4,300,966$ consecutive patients). Am J Cardiol 2008;102:1742-1746.

14 Bogdahn U, Becker G, Schlief R, Reddig J, Hassel W: Contrast-enhanced transcranial color-coded real-time sonography. Results of a phase-two study. Stroke 1993;24:676684.
15 Piscaglia F, Nolsoe C, Dietrich CF, Cosgrove DO, Gilja OH, Bachmann Nielsen $\mathrm{M}$, Albrecht T, Barozzi L, Bertolotto M, Catalano O, Claudon M, Clevert DA, Correas JM, D’Onofrio M, Drudi FM, Eyding J, Giovannini $M$, Hocke M, Ignee A, Jung EM, Klauser AS, Lassau N, Leen E, Mathis G, Saftoiu A, Seidel G, Sidhu PS, ter Haar G, Timmerman D, Weskott HP: The EFSUMB Guidelines and Recommendations on the Clinical Practice of Contrast Enhanced Ultrasound (CEUS): update 2011 on non-hepatic applications. Ultraschall Med 2012;33:33-59.

16 Touboul PJ, Hennerici MG, Meairs S, Adams $\mathrm{H}$, Amarenco P, Bornstein N, Csiba L, Desvarieux $M$, Ebrahim S, Hernandez Hernandez R, Jaff M, Kownator S, Naqvi T, Prati P, Rundek T, Sitzer M, Schminke U, Tardif JC, Taylor A, Vicaut E, Woo KS: Mannheim carotid intima-media thickness and plaque consensus (2004-2006-2011). An update on behalf of the advisory board of the 3rd, 4th and 5th watching the risk symposia, at the 13th, 15th and 20th European Stroke Conferences, Mannheim, Germany, 2004, Brussels, Belgium, 2006, and Hamburg, Germany, 2011. Cerebrovasc Dis 2012;34:290-296.

17 Rajaram V, Pandhya S, Patel S, Meyer PM, Goldin M, Feinstein MJ, Neems R, Liebson PR, Fiedler BM, Macioch JE, Feinstein SB: Role of surrogate markers in assessing patients with diabetes mellitus and the metabolic syndrome and in evaluating lipid-lowering therapy. Am J Cardiol 2004;93:32C$48 \mathrm{C}$. 
18 Saba L, Caddeo G, Sanfilippo R, Montisci R, Mallarini G: CT and ultrasound in the study of ulcerated carotid plaque compared with surgical results: potentialities and advantages of multidetector row CT angiography. AJNR Am J Neuroradiol 2007;28:1061-1066.

-19 Sitzer M, Muller W, Rademacher J, Siebler M, Hort W, Kniemeyer HW, Steinmetz H: Color-flow Doppler-assisted duplex imaging fails to detect ulceration in high-grade internal carotid artery stenosis. J Vasc Surg 1996;23: 461-465.

20 ten Kate GL, van Dijk AC, van den Oord SC, Hussain B, Verhagen HJ, Sijbrands EJ, van der Steen AF, van der Lugt A, Schinkel AF: Usefulness of contrast-enhanced ultrasound for detection of carotid plaque ulceration in patients with symptomatic carotid atherosclerosis. Am J Cardiol 2013;112:292-298.

21 McCarthy MJ, Loftus IM, Thompson MM, Jones L, London NJ, Bell PR, Naylor AR, Brindle NP: Angiogenesis and the atherosclerotic carotid plaque: an association between symptomatology and plaque morphology. J Vasc Surg 1999;30:261-268.

22 Feinstein SB: Contrast ultrasound imaging of the carotid artery vasa vasorum and atherosclerotic plaque neovascularization. J Am Coll Cardiol 2006;48:236-243.

23 Coli S, Magnoni M, Sangiorgi G, MarroccoTrischitta MM, Melisurgo G, Mauriello A, Spagnoli L, Chiesa R, Cianflone D, Maseri A: Contrast-enhanced ultrasound imaging of intraplaque neovascularization in carotid arteries: correlation with histology and plaque echogenicity. J Am Coll Cardiol 2008;52:223230.

24 Giannoni MF, Vicenzini E, Citone M, Ricciardi MC, Irace L, Laurito A, Scucchi LF, Di Piero V, Gossetti B, Mauriello A, Spagnoli LG, Lenzi GL, Valentini FB: Contrast carotid ultrasound for the detection of unstable plaques with neoangiogenesis: a pilot study. Eur J Vasc Endovasc Surg 2009;37: 722-727.

-25 Shah F, Balan P, Weinberg M, Reddy V, Neems R, Feinstein M, Dainauskas J, Meyer P, Goldin M, Feinstein SB: Contrast-enhanced ultrasound imaging of atherosclerotic carotid plaque neovascularization: a new surrogate marker of atherosclerosis? Vasc Med 2007; 12:291-297.

26 Hoogi A, Adam D, Hoffman A, Kerner H, Reisner S, Gaitini D: Carotid plaque vulnerability: quantification of neovascularization on contrast-enhanced ultrasound with histopathologic correlation. AJR Am J Roentgenol 2011;196:431-436.

27 Gronholdt ML, Nordestgaard BG, Schroeder TV, Vorstrup S, Sillesen H: Ultrasonic echolucent carotid plaques predict future strokes. Circulation 2001;104:68-73.

-28 Topakian R, King A, Kwon SU, Schaafsma A, Shipley M, Markus HS; ACES Investigators: Ultrasonic plaque echolucency and emboli signals predict stroke in asymptomatic carotid stenosis. Neurology 2011;77:751-758.
29 Vicenzini E, Giannoni MF, Puccinelli F, Ricciardi MC, Altieri M, Di Piero V, Gossetti B, Valentini FB, Lenzi GL: Detection of carotid adventitial vasa vasorum and plaque vascularization with ultrasound cadence contrast pulse sequencing technique and echo-contrast agent. Stroke 2007;38:28412843.

30 Huang PT, Huang FG, Zou CP, Sun HY, Tian XQ, Yang Y, Tang JF, Yang PL, Wang XT: Contrast-enhanced sonographic characteristics of neovascularization in carotid atherosclerotic plaques. J Clin Ultrasound 2008;36: 346-351.

- 31 Staub D, Partovi S, Schinkel AF, Coll B, Uthoff $\mathrm{H}$, Aschwanden M, Jaeger KA, Feinstein SB: Correlation of carotid artery atherosclerotic lesion echogenicity and severity at standard US with intraplaque neovascularization detected at contrast-enhanced US. Radiology 2011;258:618-626.

32 Xiong L, Deng YB, Zhu Y, Liu YN, Bi XJ: Correlation of carotid plaque neovascularization detected by using contrast-enhanced US with clinical symptoms. Radiology 2009;251:583589.

33 Staub D, Patel MB, Tibrewala A, Ludden D, Johnson M, Espinosa P, Coll B, Jaeger KA, Feinstein SB: Vasa vasorum and plaque neovascularization on contrast-enhanced carotid ultrasound imaging correlates with cardiovascular disease and past cardiovascular events. Stroke 2010;41:41-47.

- 34 Lindner JR, Coggins MP, Kaul S, Klibanov AL, Brandenburger GH, Ley K: Microbubble persistence in the microcirculation during ischemia/reperfusion and inflammation is caused by integrin- and complement-mediated adherence to activated leukocytes. Circulation 2000;101:668-675.

35 Tsutsui JM, Xie F, Cano M, Chomas J, Phillips P, Radio SJ, Lof J, Porter TR: Detection of retained microbubbles in carotid arteries with real-time low mechanical index imaging in the setting of endothelial dysfunction. J Am Coll Cardiol 2004;44:1036-1046.

36 Owen DR, Shalhoub J, Miller S, Gauthier T, Doryforou O, Davies AH, Leen EL: Inflammation within carotid atherosclerotic plaque: assessment with late-phase contrast-enhanced US. Radiology 2010;255:638-644.

-37 Shalhoub J, Monaco C, Owen DR, Gauthier T, Thapar A, Leen EL, Davies AH: Late-phase contrast-enhanced ultrasound reflects biological features of instability in human carotid atherosclerosis. Stroke 2011;42:3634-3636.

- 38 Cai J, Hatsukami TS, Ferguson MS, Kerwin WS, Saam T, Chu B, Takaya N, Polissar NL, Yuan C: In vivo quantitative measurement of intact fibrous cap and lipid-rich necrotic core size in atherosclerotic carotid plaque: comparison of high-resolution, contrast-enhanced magnetic resonance imaging and histology. Circulation 2005;112:3437-3444.

39 Hatsukami TS, Ross R, Polissar NL, Yuan C: Visualization of fibrous cap thickness and rupture in human atherosclerotic carotid plaque in vivo with high-resolution magnetic resonance imaging. Circulation 2000;102: 959-964.

-40 Kerwin W, Hooker A, Spilker M, Vicini P, Ferguson M, Hatsukami T, Yuan C: Quantitative magnetic resonance imaging analysis of neovasculature volume in carotid atherosclerotic plaque. Circulation 2003;107:851-856.

41 Saito H, Kuroda S, Hirata K, Magota K, Shiga T, Tamaki N, Yoshida D, Terae S, Nakayama $\mathrm{N}$, Houkin K: Validity of dual MRI and FFDG PET imaging in predicting vulnerable and inflamed carotid plaque. Cerebrovasc Dis 2013;35:370-377.

42 Truijman MT, Kooi ME, van Dijk AC, de Rotte AA, van der Kolk AG, Liem MI, Schreuder FH, Boersma E, Mess WH, van Oostenbrugge RJ, Koudstaal PJ, Kappelle LJ, Nederkoorn PJ, Nederveen AJ, Hendrikse J, van der Steen AF, Daemen MJ, van der Lugt A: Plaque At RISK (PARISK): prospective multicenter study to improve diagnosis of high-risk carotid plaques. Int J Stroke 2014;9: 747-754.

43 Lanza GM, Wallace KD, Scott MJ, Cacheris WP, Abendschein DR, Christy DH, Sharkey AM, Miller JG, Gaffney PJ, Wickline SA: A novel site-targeted ultrasonic contrast agent with broad biomedical application. Circulation 1996;94:3334-3340.

44 Unger EC, McCreery TP, Sweitzer RH, Shen $\mathrm{D}, \mathrm{Wu} \mathrm{G}$ : In vitro studies of a new thrombusspecific ultrasound contrast agent. Am J Cardiol 1998;81:58G-61G.

45 Kaufmann BA, Carr CL, Belcik JT, Xie A, Yue Q, Chadderdon S, Caplan ES, Khangura J, Bullens S, Bunting S, Lindner JR: Molecular imaging of the initial inflammatory response in atherosclerosis: implications for early detection of disease. Arterioscler Thromb Vasc Biol 2010;30:54-59.

46 Khanicheh E, Mitterhuber M, Xu L, Haeuselmann SP, Kuster GM, Kaufmann BA: Noninvasive ultrasound molecular imaging of the effect of statins on endothelial inflammatory phenotype in early atherosclerosis. PLoS One 2013;8:e58761.

47 Chadderdon SM, Belcik JT, Bader L, Kirigiti MA, Peters DM, Kievit P, Grove KL, Lindner JR: Proinflammatory endothelial activation detected by molecular imaging in obese nonhuman primates coincides with onset of insulin resistance and progressively increases with duration of insulin resistance. Circulation 2014;129:471-478.

48 Yang H, Zhang L, Xiong X, Liu Y: Specific adhesion and accumulation of VCAM-1-targeted ultrasound microbubbles to inflammatory endothelial cells under hemodynamic shear flow simulation. J Control Release 2011; 152(suppl 1):e227-e229.

49 Kuliszewski MA, Fujii H, Liao C, Smith AH, Xie A, Lindner JR, Leong-Poi H: Molecular imaging of endothelial progenitor cell engraftment using contrast-enhanced ultrasound and targeted microbubbles. Cardiovasc Res 2009;83:653-662. 
50 Liu H, Wang X, Tan KB, Liu P, Zhuo ZX, Liu Z, Hua X, Zhuo QQ, Xia HM, Gao YH: Molecular imaging of vulnerable plaques in rabbits using contrast-enhanced ultrasound targeting to vascular endothelial growth factor receptor-2. J Clin Ultrasound 2011;39:8390.

51 McCarty OJ, Conley RB, Shentu W, Tormoen GW, Zha D, Xie A, Qi Y, Zhao Y, Carr C, Belcik T, Keene DR, de Groot PG, Lindner JR: Molecular imaging of activated von willebrand factor to detect high-risk atherosclerotic phenotype. JACC Cardiovasc Imaging 2010;3:947-955.
52 Schumann PA, Christiansen JP, Quigley RM, McCreery TP, Sweitzer RH, Unger EC, Lindner JR, Matsunaga TO: Targeted-microbubble binding selectively to GPIIb IIIa receptors of platelet thrombi. Invest Radiol 2002;37:587-593.

53 Alonso A, Della Martina A, Stroick M, Fatar M, Griebe M, Pochon S, Schneider M, Hennerici M, Allemann E, Meairs S: Molecular imaging of human thrombus with novel abciximab immunobubbles and ultrasound. Stroke 2007;38:1508-1514.

54 Takeuchi M, Ogunyankin K, Pandian NG, McCreery TP, Sweitzer RH, Caldwell VE, Unger EC, Avelar E, Sheahan M, Connolly R: Enhanced visualization of intravascular and left atrial appendage thrombus with the use of a thrombus-targeting ultrasonographic contrast agent (MRX-408A1): in vivo experimental echocardiographic studies. J Am Soc Echocardiogr 1999;12:1015-1021.
55 Wang X, Hagemeyer CE, Hohmann JD, Leitner E, Armstrong PC, Jia F, Olschewski M, Needles A, Peter K, Ahrens I: Novel singlechain antibody-targeted microbubbles for molecular ultrasound imaging of thrombosis: validation of a unique noninvasive method for rapid and sensitive detection of thrombi and monitoring of success or failure of thrombolysis in mice. Circulation 2012;125:3117-3126.

$56 \mathrm{Wu}$ W, Wang Y, Shen S, Wu J, Guo S, Su L, Hou F, Wang Z, Liao Y, Bin J: In vivo ultrasound molecular imaging of inflammatory thrombosis in arteries with cyclic Arg-Gly-Aspmodified microbubbles targeted to glycoprotein IIb/IIIa. Invest Radiol 2013;48:803-812. 